\title{
Research Paper \\ The Comparison of the Effects of Voluntary Running Wheel and Endurance Training on II-6 and Testosterone Levels in the Rats With PCOS
}

\author{
Faranak Moradi ${ }^{1}$ (), Ali Akbarnejad² (1)
}

1. Department of Sport Physiology, Faculty of Physical Education, University of Al Zahra, Tehran, Iran.

2. Department of Physiology, Faculty of Physical Education, Tehran University, Tehran, Iran.

\begin{tabular}{|c|c|}
\hline $\begin{array}{l}\text { Use your device to scan } \\
\text { and read the article online }\end{array}$ & Citation: Moradi F. Akbarnejad A. [The Comparison of the Effects of Voluntary Running Wheel and Endurance Training \\
\hline Dritg & $\begin{array}{l}\text { Il-6 and Testosterone Levels in the Rats With PCOS (Persian)]. Journal of Arak University of Medical Sciences (JAMS). 2021; } \\
\text { 24(3):412-423. https://doi.org/10.32598/JAMS.24.3.6212.1 }\end{array}$ \\
\hline atros & doi'https://doi.org/10.32598/JAMS.24.3.6212.1 \\
\hline
\end{tabular}

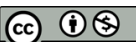

Article Info:

Received: 07 Jun 2020

Accepted: 23 May 2021

Available Online: 01 Aug 2021

Key words:

IL-6, PCOS, Testos-

terone, Endurance

Training, Voluntary

Training

\section{A B STRACT}

Background and Aim Polycystic Ovary Syndrome (PCOS) is an endocrine disorder in women of reproductive age. In cross-sectional and longitudinal studies, exercise training is an effective solution for treating and declining the symptoms. This study aims to compare the effects of the voluntary and the endurance exercise on some of the inflammatory and hormonal markers in rats with PCOS.

Methods \& Materials In this study, Which was experimental and applied in terms of purpose, 40 female Wistar rats with Mean \pm SD weight of $220 \pm 20$ g were divided randomly into four groups of ten: 1 ) control, 2) PCOS, 3) PCOS + Running wheel and 4) PCOS + endurance training. The rats in the voluntary group experienced voluntary activity of wheel running for 8 weeks ( 5 times a week). Alternatively, the endurance training group underwent aerobic exercises for 8 weeks ( 5 sessions in a week) on a treadmill. The blood samples were taken from the heart to measure the concentration of interleukin- 6 and testosterone level and were analyzed by one-way analysis of variance and LSD post hoc test.

Ethical Considerations This study was approved by the Tehran University, Iran (Code: IR.UT.REC.1395016). Results The results showed no significant difference in IL-6 and testosterone indices between endurance and voluntary training groups $(P=0.565),(P=0.496)$. Both training groups (endurance and voluntary) separately resulted in a significant reduction in il6 and testosterone compared to rats with $P \operatorname{COS}(P=0.002)$, $(P=0.000)$, $(P=0.038),(P=0.007)$.

Conclusion Altogether, the present study's findings suggest that voluntary and endurance training in rats have similar effects on IL-6, Testosterone, and weight.

\section{Extended Abstract}

\section{Introduction}

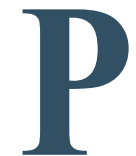

olycystic Ovary Syndrome (PCOS) is an endocrine disorder in women of reproductive age. . Chronic inflammatory condition is seen in most women [1]. Interleukin-6 is one of the inflammatory factors that sever- al studies have shown to increase its levels in patients with PCOS [2]. In addition, one of the problems with PCOS is the imbalance of secretion of hormones as a result of blood androgen levels, including testosterone is higher in people with this syndrome than in ordinary people [4], which leads to a decrease in the ability to conceive and hair growth on the face and body [5]. In cross-sectional and longitudinal studies, exercise training is an effective solution for treating and declining the symptoms. This study aimed to compare

\section{* Corresponding Author:}

Ali Akbarnejad, PhD.

Address: Department of Physiology, Faculty of Physical Education, Tehran University, Tehran, Iran.

Tel: +98 (912) 0760472

E-mail: aakbarnejad@ut.ac.ir 
Table 1. The effect of voluntary and endurance training on rat weight

\begin{tabular}{ccccc}
\hline Groups & Control & PCOS & Endurance Training & Voluntary Training \\
\hline The weight of rats & $239.8 \pm 2.48$ & $255 \pm 2.99$ & $210.3 \pm 1.87$ & $213.7 \pm 1.71$ \\
\hline & & & S \\
& & & Sournal of \\
& & & Arak University of Medical Sciences
\end{tabular}

the effects of the voluntary and the endurance exercise on inflammatory and hormonal markers in rats with PCOS.

\section{Materials and Methods}

In this purposive study, 40 female Wistar rats with a Mean \pm SD weight of $220 \pm 20 \mathrm{~g}$ were divided randomly into four groups of ten: 1) control, 2) PCOS, 3) PCOS + Running wheel, and 4) PCOS + endurance training. To induce the phenotype of polycystic ovary syndrome, hormonal induction of dissolved estradiol valerate was used in every 4 $\mathrm{g}$ of body weight, $4 \mathrm{mg}$ in $2 \mathrm{ml}$ of olive oil [16]. The rats in voluntary group experienced voluntary activity of wheel running for 8 week ( 5 times a week). Alternatively, the endurance training group, underwent aerobic exercises for 8 weeks ( 5 sessions in a week) on a treadmill. Rats were anesthetized in accordance with ethical principles and according to the animal care laws of Iran (initially using a mixture of ketamine $30-50 \mathrm{mg} / \mathrm{kg}$ body weight, intraperitoneally) and xylazine ( 3 to $5 \mathrm{mg} / \mathrm{kg}$ body weight, intraperitoneal) [19].Then the blood samples were taken from heart to measure the concentration of interleukin- 6 and testosterone level and were analyzed by one-way analysis of variance and LSD post hoc test [15-18].

\section{Results}

According to the findings (Table 1), after the end of 8 weeks of training, the weight of rats in the training groups (endurance and voluntary) decreased compared to the group with PCOS, which was not statistically significant, and also the rats' weight in the PCOS group. It increased in the control and experimental groups, but this increase was not significant $(\mathrm{P}<0.05)$.

The one-way analysis of variance showed a significant difference between IL- 6 and testosterone levels ( $\mathrm{P}=0.000)$ $(\mathrm{P}=0.039)$. To determine the exact difference between the groups, the LSD post hoc test was used, which showed no significant difference between the groups of endurance training and voluntary training $(\mathrm{P}=0.565)(\mathrm{P}=0.496)$. However, both exercises separately significantly reduced IL-6 and testosterone in trained rats compared to polycystic rats $(\mathrm{P}=0.002),(\mathrm{P}=0.000),(\mathrm{P}=0.038),(\mathrm{P}=0.007)$ (Figure 1).

\section{Discussion and Conclusion}

Findings showed no significant difference between the effect of 8 weeks of voluntary training on the wheel and endurance training on IL-6 and testosterone levels in rats with polycystic ovary syndrome $(\mathrm{P}=0.565),(\mathrm{P}=0.496)$.

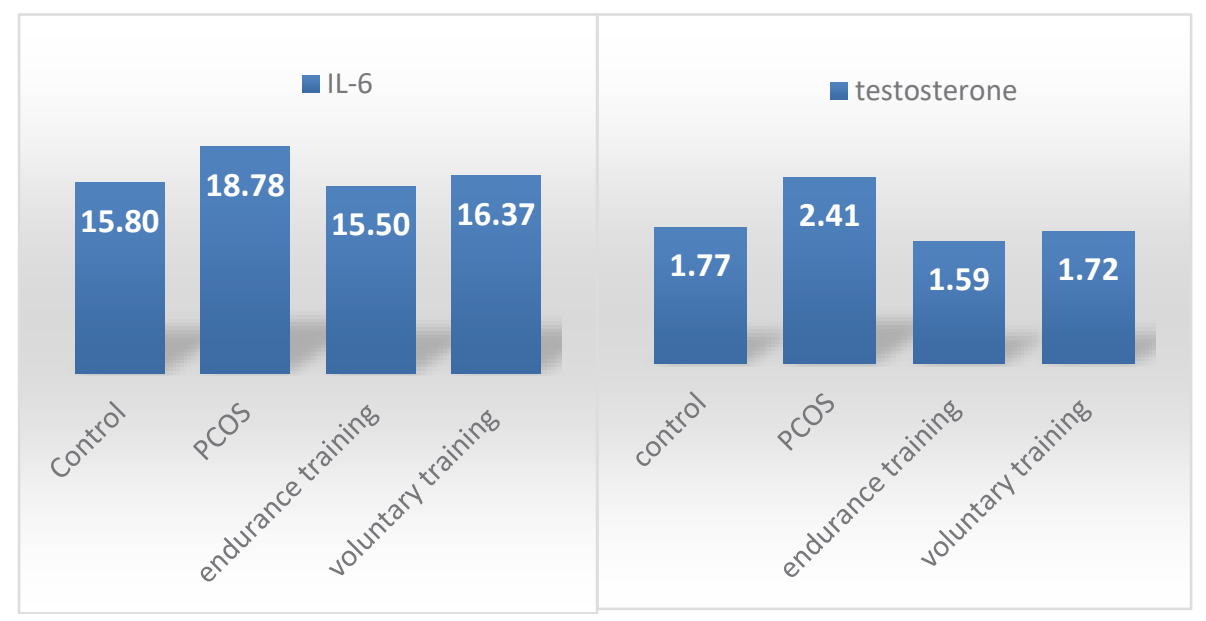

Figure 1. IL-6 and testosterone levels in 4 groups after the exercise protocol 
However, both training groups (endurance and voluntary) separately led to a significant decrease in IL-6 and Testosterone compared to rats with $\mathrm{PCOS}(\mathrm{P}=0.002)$, $(\mathrm{P}=0.000),(\mathrm{P}=0.038),(\mathrm{P}=0.007)$.

As a result of intense endurance exercise, the athlete's body suffers from physiological inflammation caused by immune responses for some time [22]. Research has shown that exercise, especially if performed with high intensity or duration, leads to the release of many cytokines into the bloodstream [23]. Following the physical activity, the production of inflammatory cytokines such as tumor necrosis factor, interleukin, and acute-phase reactive protein increases briefly [22]. Still, the researchers' findings suggest that these indices decrease favorably as a result. Performing a period of regular endurance activities [24].

Overall, the results showed that endurance training compared to voluntary training had the same effect on serum IL-6 and testosterone levels, and both reduced the levels of these two factors. As a result, regular and not too heavy exercise (voluntary and endurance) for these patients, apart from clinical treatments, is a healthy and natural way to improve polycystic ovary syndrome.

\section{Ethical Considerations}

\section{Compliance with ethical guidelines}

In this study, all experimental protocols on laboratory animals were performed according to the instructions of the Ethics Committee for Working with Laboratory Animals, University of Tehran, by assigning the ethics code IR.UT. REC.1395016, and the standards of the principles of working with laboratory animals were observed.

\section{Funding}

This research did not receive any grant from funding agencies in the public, commercial, or non-profit sectors.

\section{Authors' contributions}

All authors equally contributed to preparing this article.

\section{Conflicts of interest}

The authors declared no conflicts of interest.

\section{Acknowledgements}

The authors would like to thank the esteemed officials and staff of the Faculty of Physical Education, University of Tehran, for preparing the present project. 


\section{مقايسه اثر دو نوع تمرين اختيارى و استقامتى بر سطوح ايثترلوكين 7 و تستوسترون در موش هاى

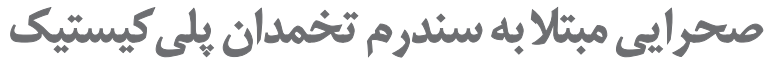

فرانك مرادى' ه. "على اكبرنزاد'

ا. بروه فيزيولورى ورزشى، دانشكده تربيتبدنى، دانشعاه الزهراء تهرانه ايران.

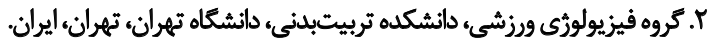

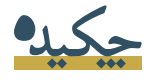

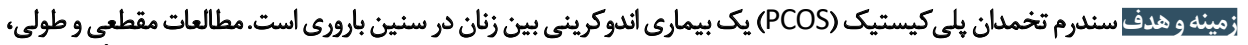

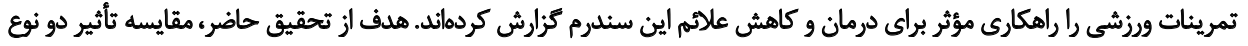

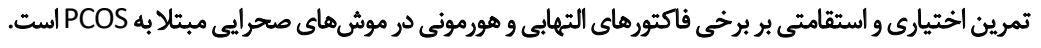

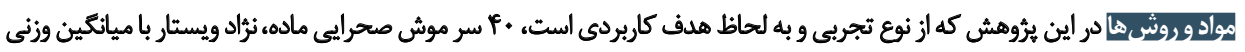

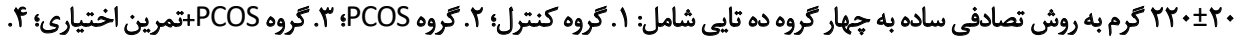

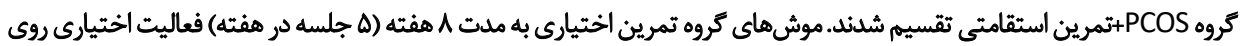

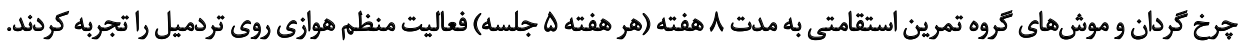

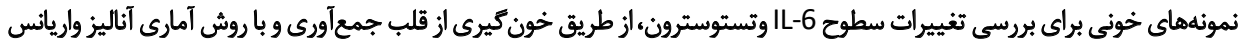

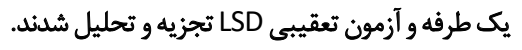

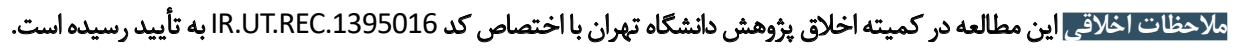

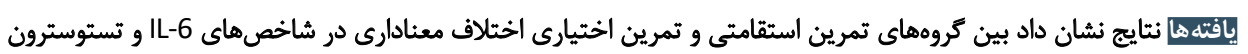

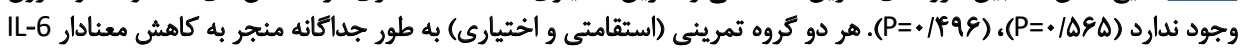

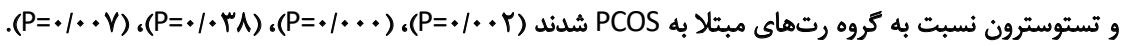

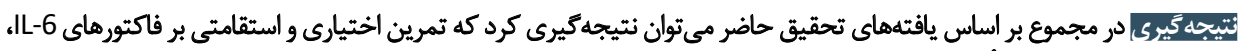

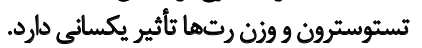

اطلاعات مقاله:

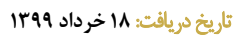

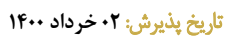

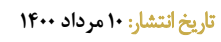

كليدواثرها:

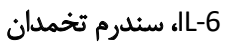

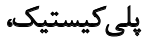
تستوسترون، تمرين استقامتى، تمرين اختيارى استقامئ تم

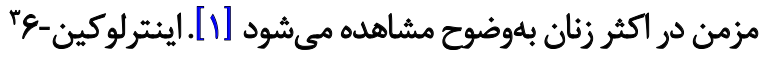

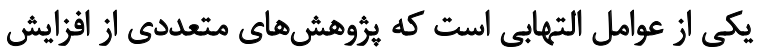

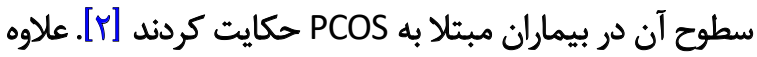

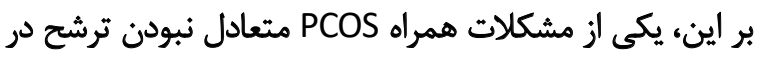

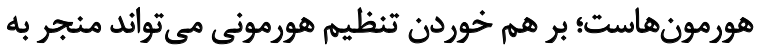

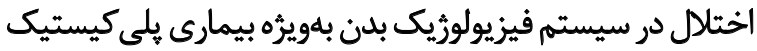

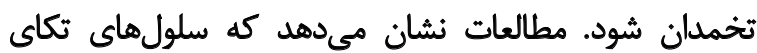

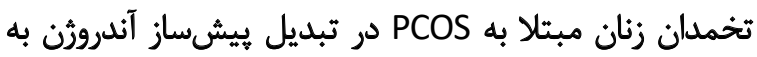

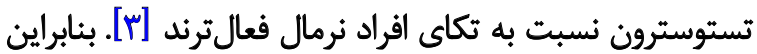

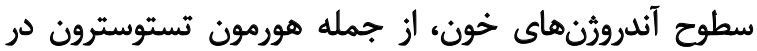

استاين و لونتال كسانى بودند كه براى اولين بار از سندرم

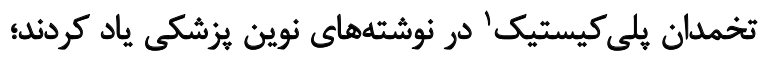

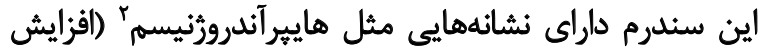

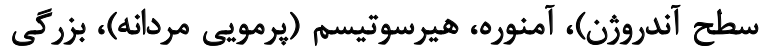

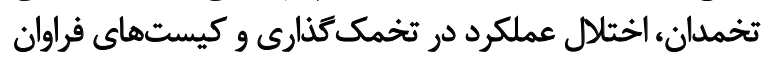
تخمدان است. در سندرم تخمدان يلم كيستيك وضعيت التهابى تخيى

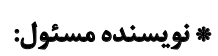

دكتر على اكبرنزئراد

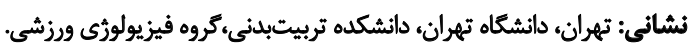
تلفن: يست الكترونيكي: بakbarnejad@ut.ac.ir 
است با نبود تغيير و يا كاهش مقادير استراحتى تستوسترون

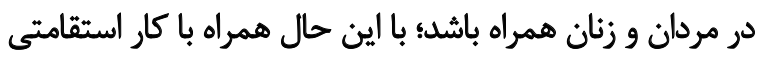

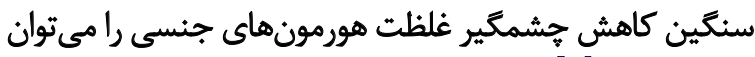

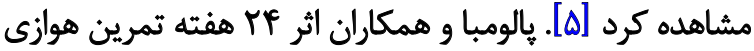

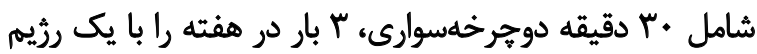

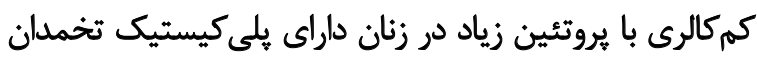

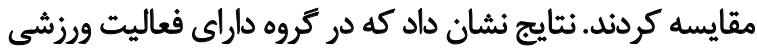

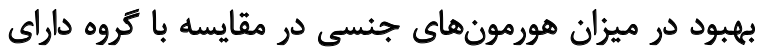

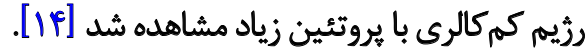

كاهى انجام شدتهاى كوناگون تمرين براى اين بيمارئ

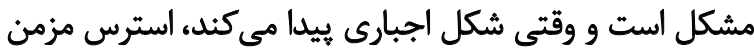

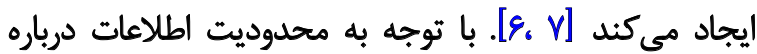

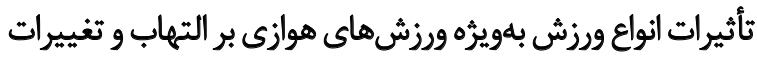

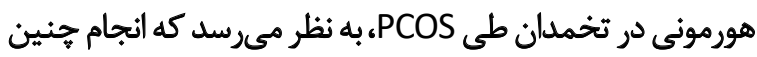

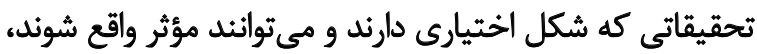

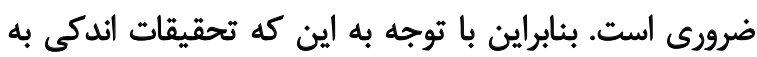

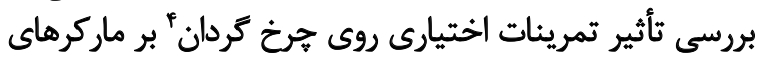

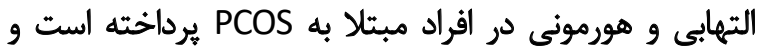

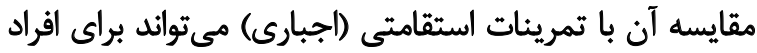

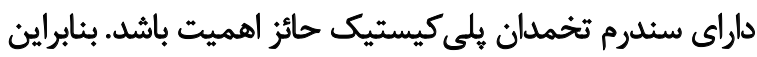

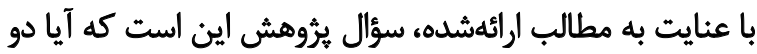

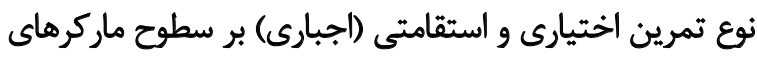

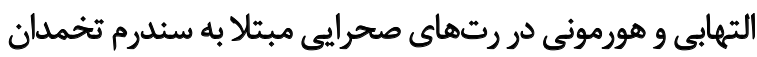
هلى كيستيك تأثير دارد ياخير؟

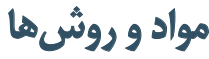

تحقيق حاضر به روش تجربى در سال ذهوبا در آزمايشكاه

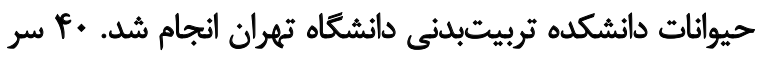

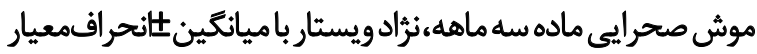

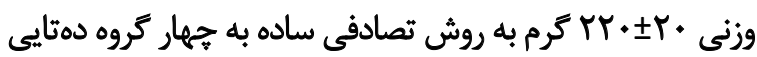

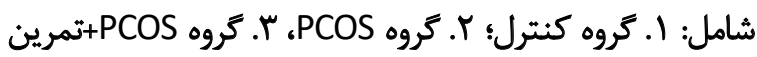

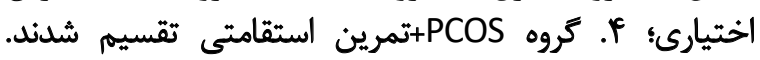

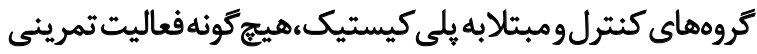

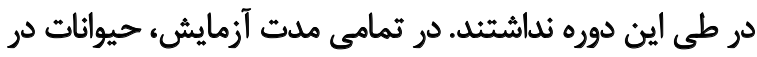

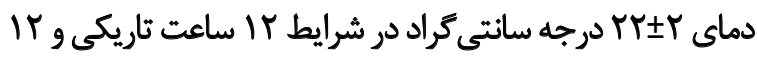

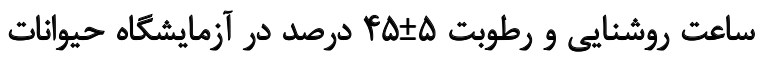

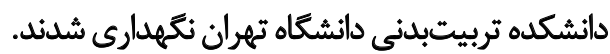

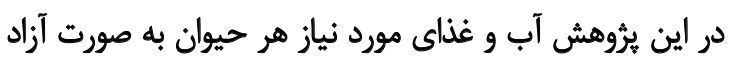

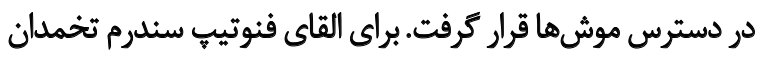

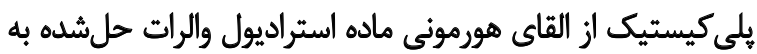

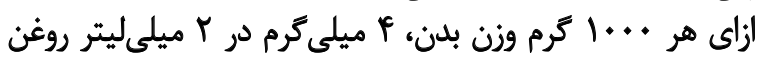

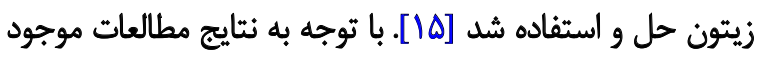

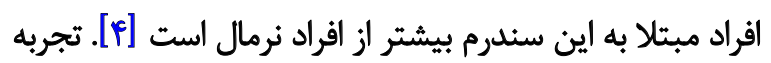

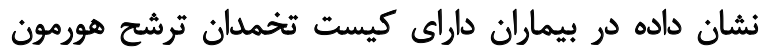

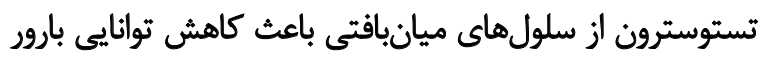

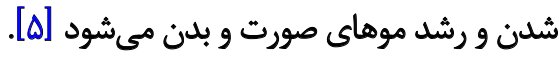
روند رو به افزايش ابتلا به PCOS در دنيا و اثرات نامطلوب

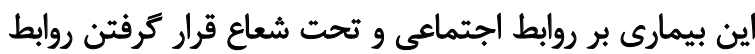

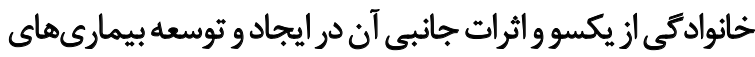

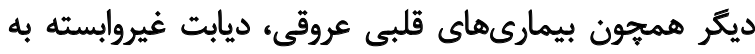

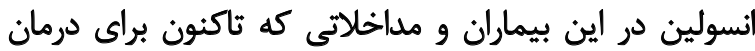

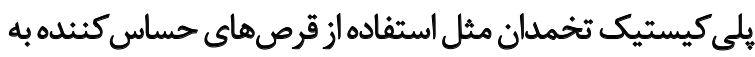

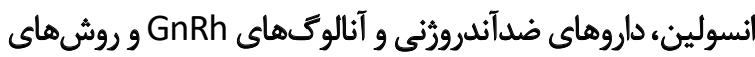

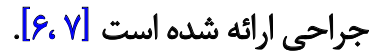

علىرغم درمانهاى در دسترس، هيج مداخله فارماكولوريك

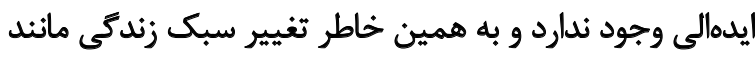

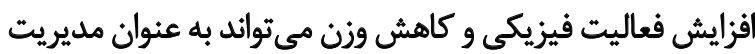

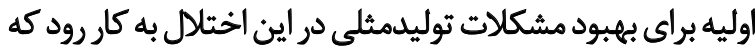

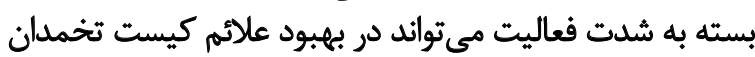

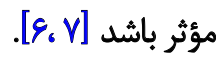

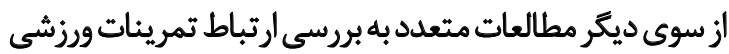

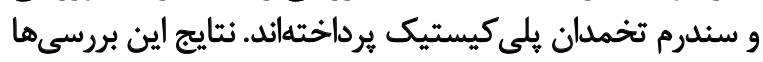

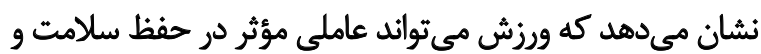

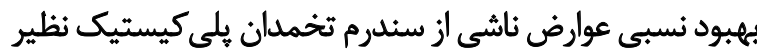

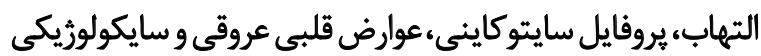

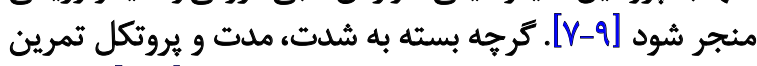

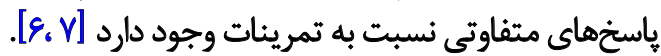
در ياسخ به ورزش، اينترلوكين-9 بيشتر از هر سايتوكاين

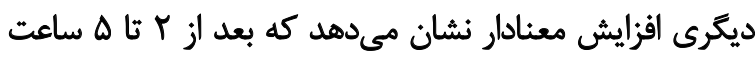

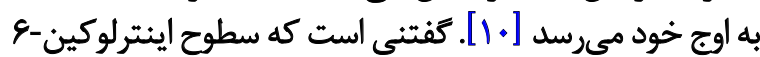

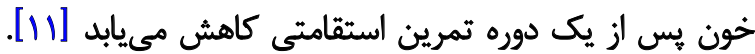

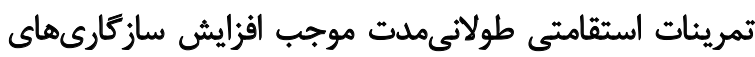

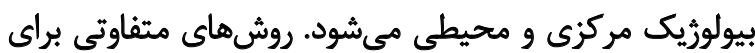

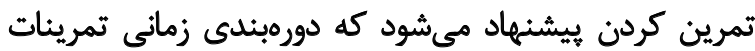

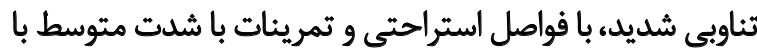

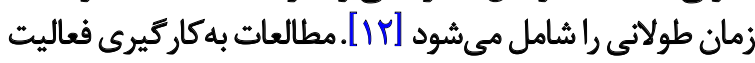

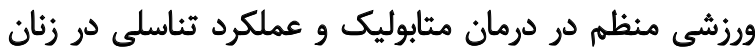

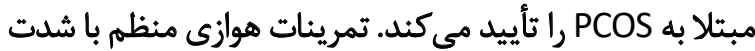

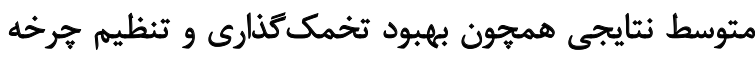

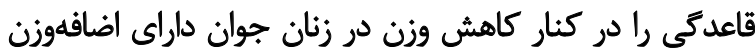

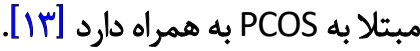

شايان ذكر است مقدار تستوسترون استراحتى، معمولاً در

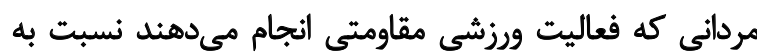
مقدار آن در ساير افراد بيشتر است اما تمرينات استقامتى ممكن 
جدول ا. يروتكل تمرين

\begin{tabular}{|c|c|c|c|c|c|c|c|c|}
\hline هشتم & هفتم & شُشم & ينجمي & حهلارم & سوم & دوم & اول & هفته \\
\hline 8. & q. & e. & $\Delta \Delta$ & $\Delta$. & r. & $r$. & 10 & ملت (دقيقه) \\
\hline r. & r. & r. & rA & TQ & r. & 10 & 1. & سرعت (m/min) \\
\hline
\end{tabular}

الكتريكى استفاده شد. يس از دو هفته بلهتديج هر دو هفته شدت

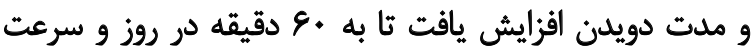

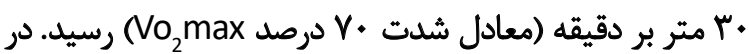
كل برنامه تمرينى شيب تردميل ثابت بود (صفر درجه). برنامه دئه

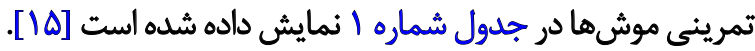

\section{ig 5 signi}

براى تعيين مقادير متغيرهاي آزمون، رتها با رعايت اصول

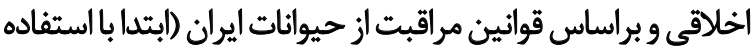

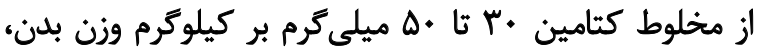

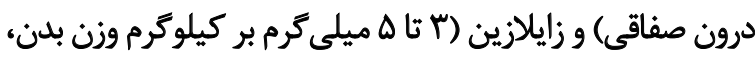

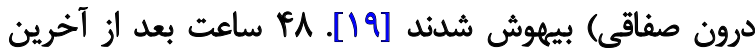

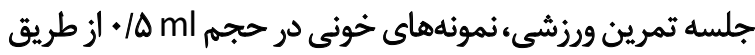

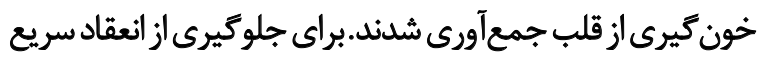

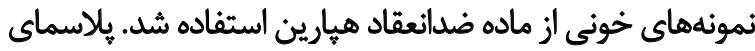

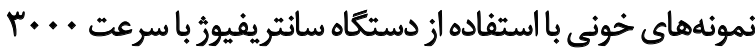

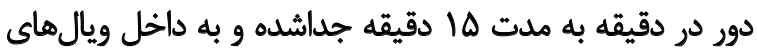

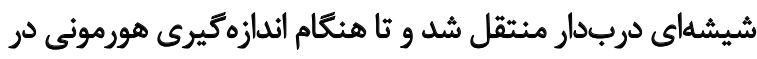

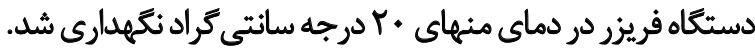

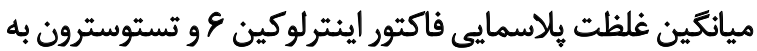
ترتيب از روش راديوايمنواسي با استفاده از دستكاه خوانئده الايزا

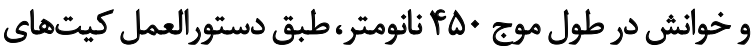

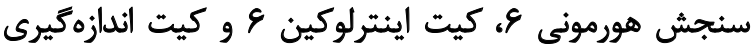

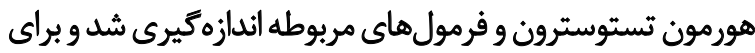
تجزيه و تحليل آمارى از آن استفاده شُوند.

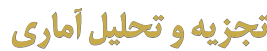

دادههاي بهدست آمده براي مقايسه ميانكين تغييرات بانرمافزار ورئ · TPSS

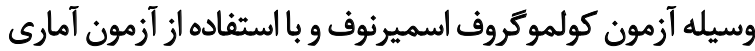

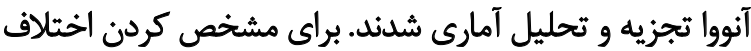

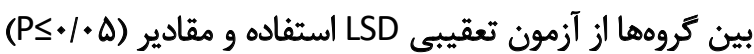

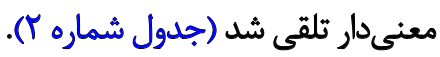

به دليل بروسي اثر شدت و نوع تمرين بر بهبود علائم دCOS

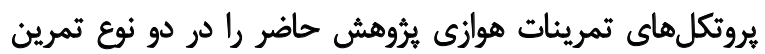

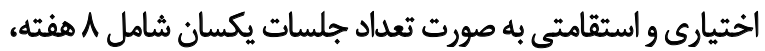

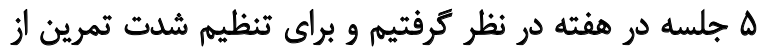

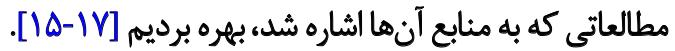

\section{(Running wheel) بروتكل ثمرين أفتشياوى}

موشهاي كروه تمرين برنامه آشنايي با تمرين شُامل راه رفتن

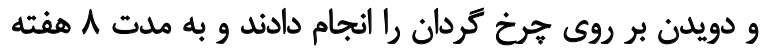

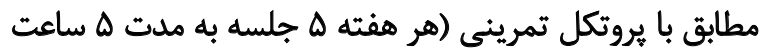

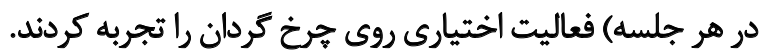

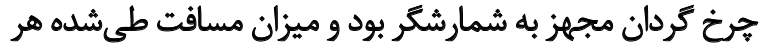

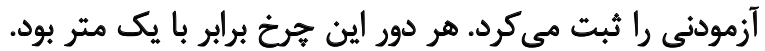

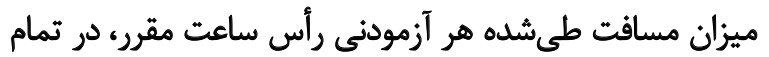

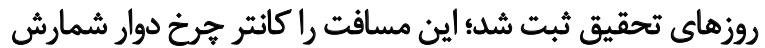

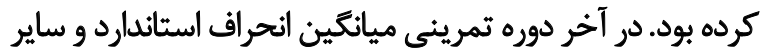

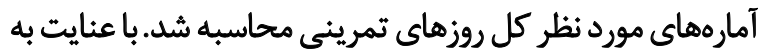

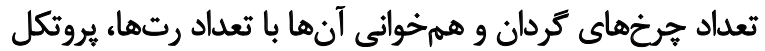

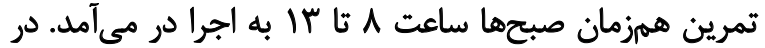

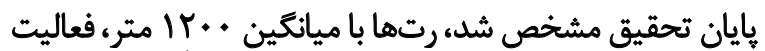

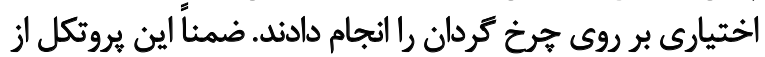

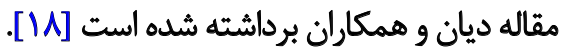

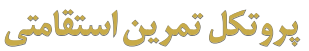

موش هاي كروه تمرين استقامتي برنامه آشنايى با تمرين شامل

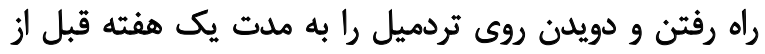

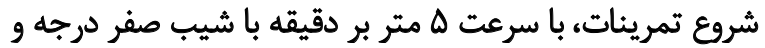

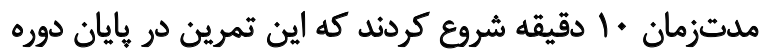

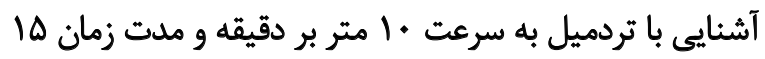

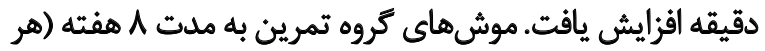

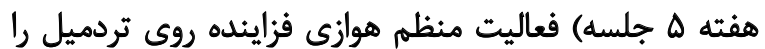

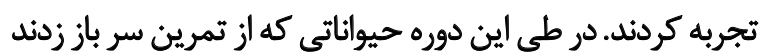

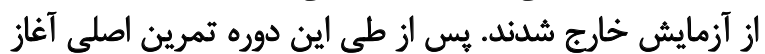

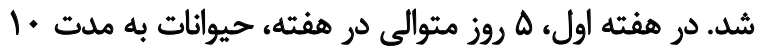

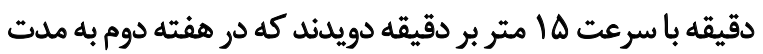

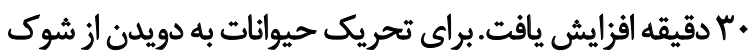


جدول r. مقادير متغيرهاى يُؤهش در جهار كروه يس از انجام يروتكل تمرين

\begin{tabular}{|c|c|c|c|c|}
\hline \multicolumn{4}{|c|}{ ميانكين +انحرافمعيار } & \multirow{2}{*}{ كروهها } \\
\hline تمرين اختيارى & تمرين استقامتى & Pcos & كتترل & \\
\hline $19 / Y Y \pm V / \Delta T Y$ & $|\Delta / \Delta \cdot \pm 1 / V 9|$ & 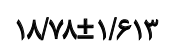 & $\mid \Delta / \Lambda \pm \pm V / r / T$ & IL-6 (pg/ml) \\
\hline $1 / V / \Delta \pm \cdot / \Delta 1 \Delta$ & $V / \Delta q \pm \cdot / \Delta \cdot \varphi$ & $r / P \backslash \pm 1 \Delta / u Y$ & $1 / W \pm \cdot / \pi T$ & تستوسترون (ng/ml) \\
\hline
\end{tabular}

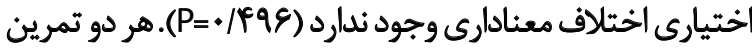

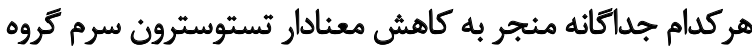

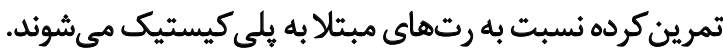

بحث

يافتهاى تحقيق نشان دادكه بين ثأثير 1 هفته تمرين

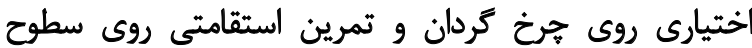

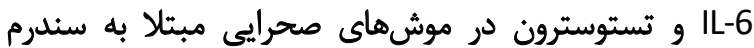

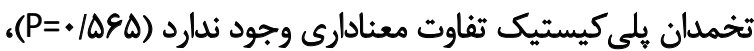

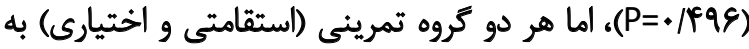

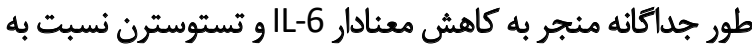
كروه رتهاى مبتلا به PCOS شدانه

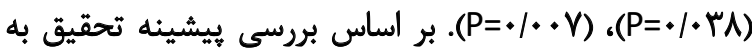

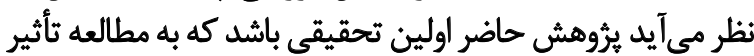

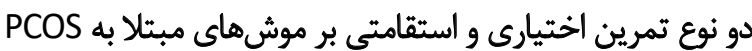

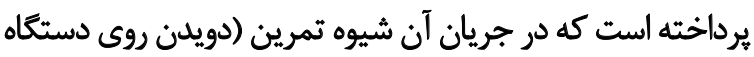

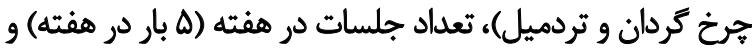

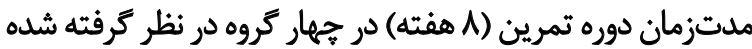

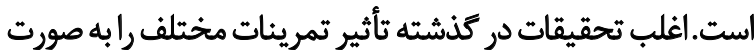

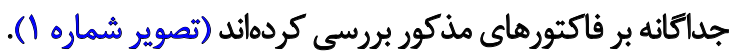
يافتهاى تحقيق حاضر درباره تفاوت نداشتن هر دو نوع

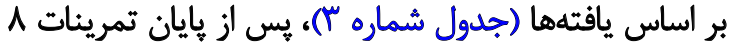

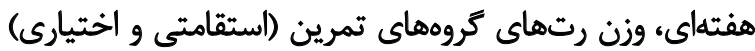

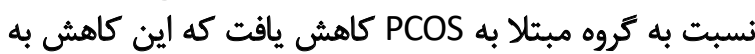

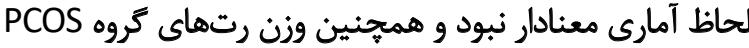

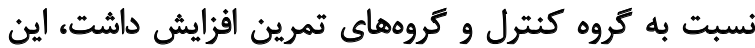

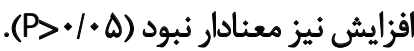

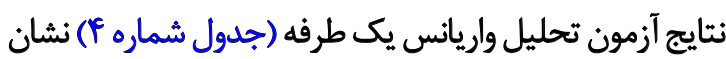

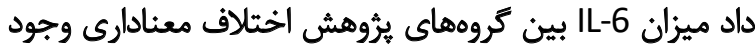

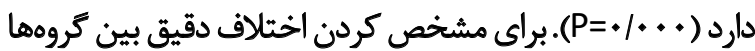

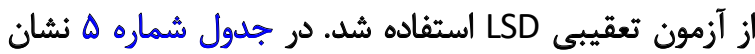

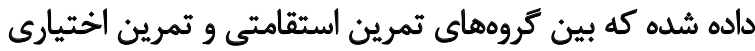

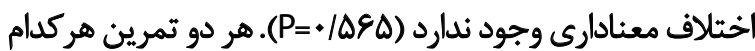

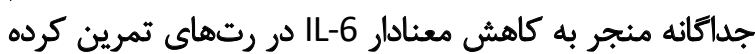

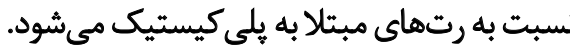
نتايج آزمون تحليل واريانس يك طرفه در ميزان تستوسترون

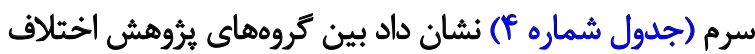

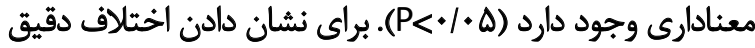

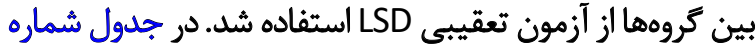

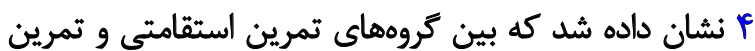

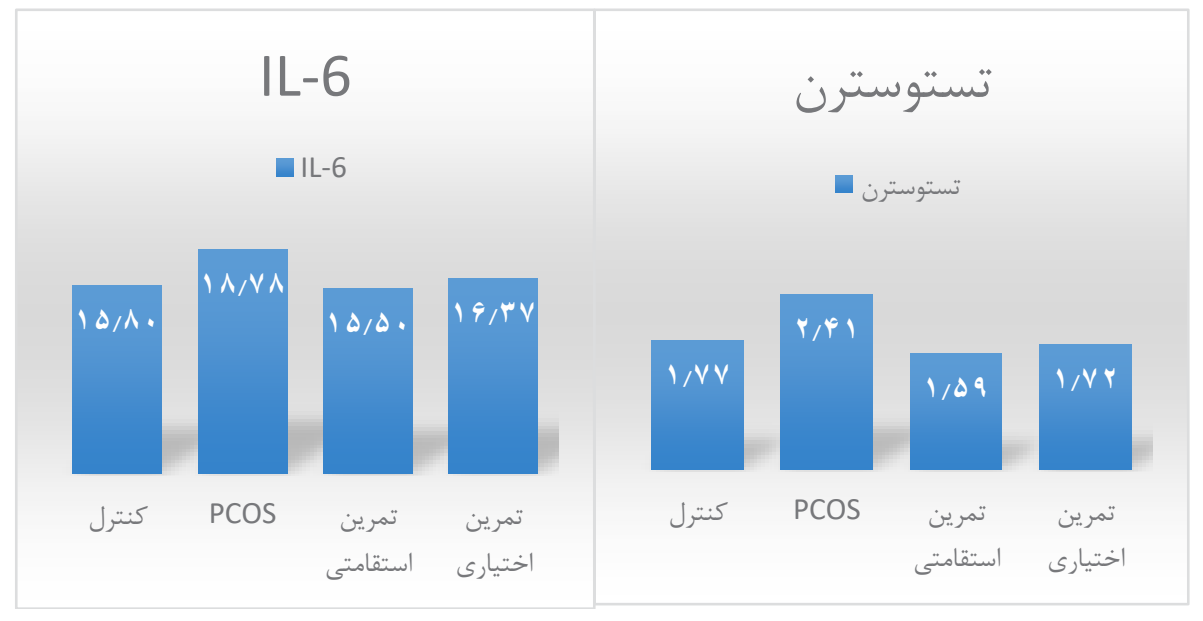


جدول ץ. تأثير تمرين اختيارى و استقامتى بر وزن رتها

\begin{tabular}{|c|c|c|c|c|}
\hline تمرين اختيارى & تمرين اسثقامتى & PCOS & كتترل & S \\
\hline$r \mid r / V \pm I / M$ & $r 1+/ \mu \pm 1 / A V$ & $r \Delta \Delta \pm r / a q$ & $r+q / \lambda \pm r / F \lambda$ & وزن بلن رتها \\
\hline
\end{tabular}

تمرين استقامتي نوعي تمرين است كه با حجم زياد و شدت

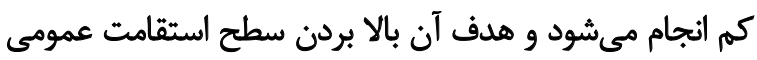

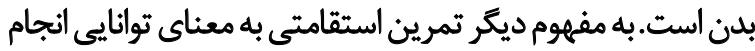

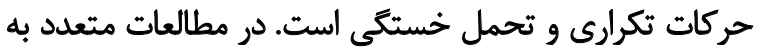

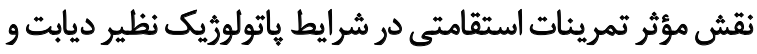

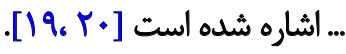

نتايج مطالعه كيلاني و همكاران نشان داد كه انجام 1 هفته تمرينات منظم استقامتى در خانمهاى مبتلا به سندرم تخمدان

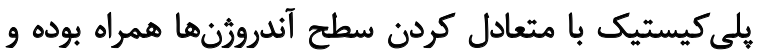

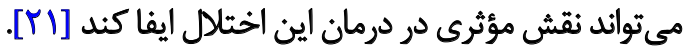

در اثر تمرينات ورزشي استقامتي شديلد، بلن ورزشكار براى مدتي

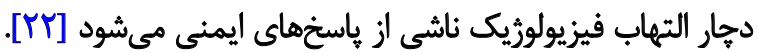

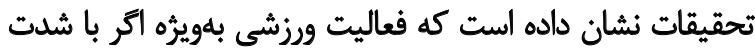
يا مدت زياد انجام شود، منجر به آزادسازى بسيارى ازئ إن سايتوكينها

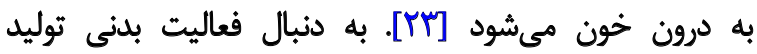

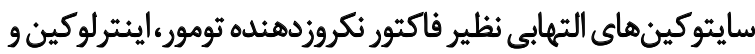

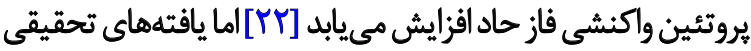

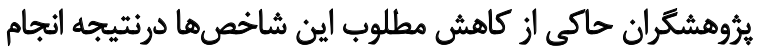

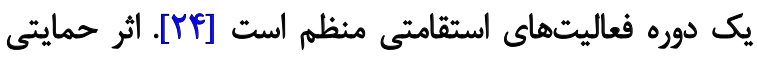

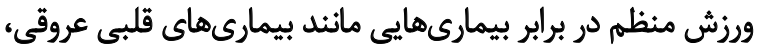

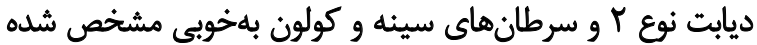

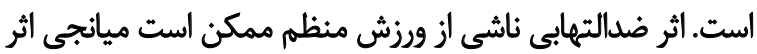

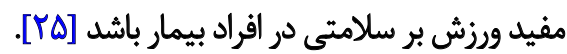

تمرين در كاهش فاكتور التهابى 6-6 دادر رتهاي مبتلا به سندرم تخمدان ثلى كيستيك، با نتايج يافتههاي دريانوش و و همكاران

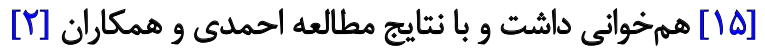

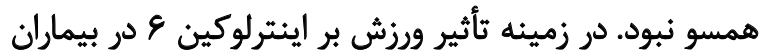

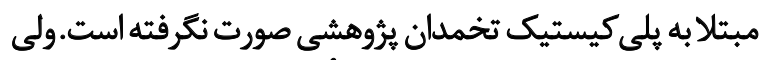

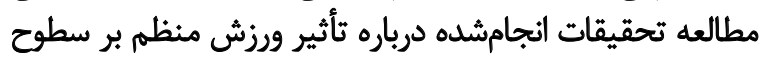

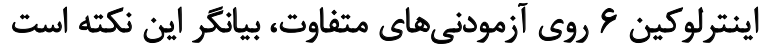

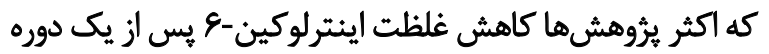

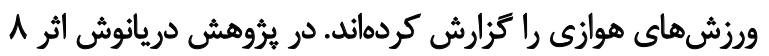

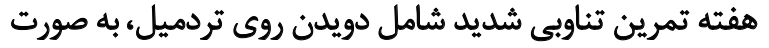

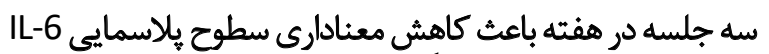

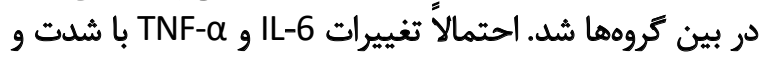

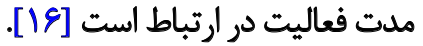

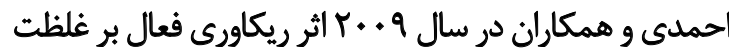

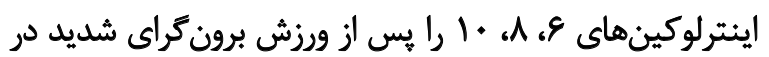

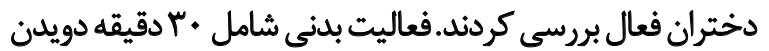

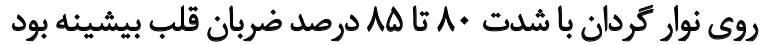

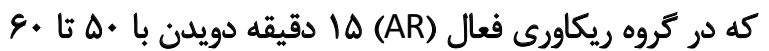

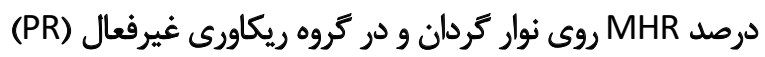

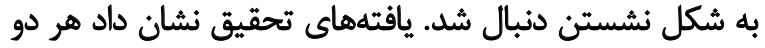

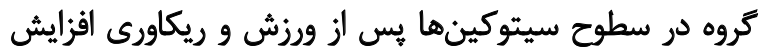

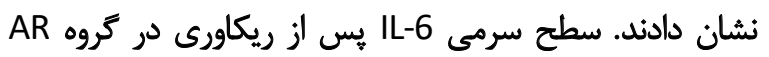

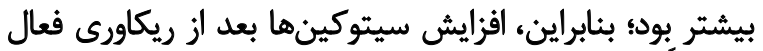

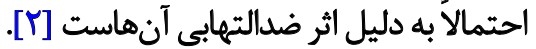

جدول F. نتايج آزمون تحليل واريانس يكطرفه براى ثأثير تمرين استقامتى و اختيارى بر IL-6 و تستوسترون

\begin{tabular}{|c|c|c|c|c|c|c|}
\hline $\mathbf{P}$ & $\mathbf{F}$ & ميانكين مربعات & درجه أزادى & مجموع مربعات & & \\
\hline \multirow[t]{3}{*}{$+\%$} & $q / r^{\prime}$ & $\mid \& / \Lambda \Delta \Delta$ & $r$ & $\Delta \cdot / \Delta \& \Delta$ & بين كروهي & \\
\hline & & $1 / A I A$ & re & gA/RTT & درون كروهي & $\begin{array}{c}\text { IL-6 } \\
\mathrm{pg} / \mathrm{ml}\end{array}$ \\
\hline & & & rq & $110 / 94$ & كل & \\
\hline \multirow[t]{3}{*}{$.1 .+9$} & $r / .9 r$ & I/rVq & $r$ & r/ArA & يين كروهيى & \\
\hline & & Pif & re & |P/Aq & درونكروهى & تستوسترون \\
\hline & & & rq & IV/MTE & كل & \\
\hline
\end{tabular}


جدول ه. نتايج آزمون تعقيبى LSD براي مقايسه IL-6 و تستوسترون در كروهماى يثروهش

\begin{tabular}{|c|c|c|c|c|c|}
\hline $\mathbf{P}$ & خطاى استاندارد & ميانيكين اختالف & ئروه & يروه تمرين & \\
\hline$. / Y \Delta \mid$ & .18 & $. / \Delta V+\ldots$. & كنترل & & \multirow{6}{*}{ IL-6 (pg/ml) } \\
\hline .1. & .18 & $-r / F \mid \ldots+$. & Pcos & استقامتى & \\
\hline - /AFA & .18. & $-. / 4 \Delta \cdot \ldots .$. & اختيارى & & \\
\hline - Ire & .18 & $.197 \ldots . .$. & كنترل & \multirow{3}{*}{ اختيارى } & \\
\hline.$/ \Delta F \Delta$ & .18 &.$/ T \Delta \ldots$ & استقامتي & & \\
\hline $.1 \cdot \cdot r$ & .18 & $-r / . \varepsilon \ldots \ldots$ & PCOS & & \\
\hline . LAPY &.$/ K A$ &.$- / 1 \mathrm{~W} \cdot \ldots$ & كتترل & \multirow{3}{*}{ استقامتى } & \multirow{6}{*}{ تستوسترون (ng/ml) } \\
\hline$\% v$ & $\cdot / T A$ & $-\bullet / \Lambda \mid \Lambda \cdot \cdots \cdot \cdot$ & PCOS & & \\
\hline.$/ 49$ & $\cdot / R$ & $-+/ 14 \ldots \ldots$ & اختيارى & & \\
\hline . /apt & $+/ \pi \Lambda$ & $. / . r I \ldots . .$. & كنترل & \multirow{3}{*}{ اختيارى } & \\
\hline.$/ F q$ & $\cdot / M A$ & $. / 194 \ldots+$. & استقامتى & & \\
\hline$\%$ &.$/ K A$ & -./er..... & PCOS & & \\
\hline
\end{tabular}

جلسه در هفته و هر جلسه به مدت •9 دقيقه روى هورمونهاى

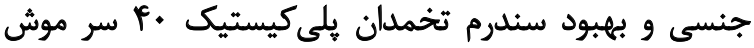

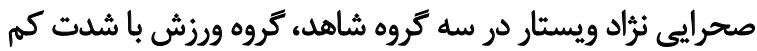

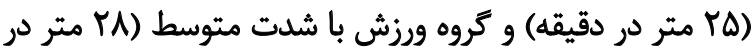

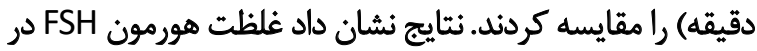

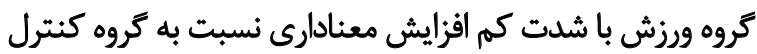

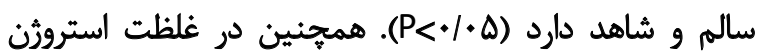

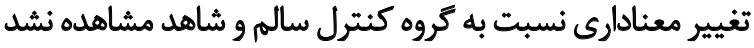

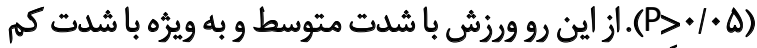

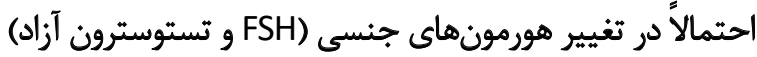

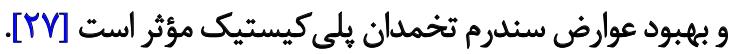

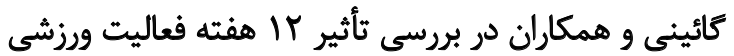

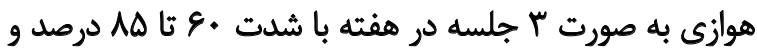

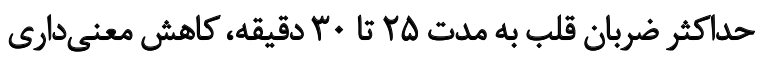

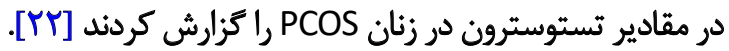

در توضيح سازوكار كاهش تستوسترون مىتوان اينكونه

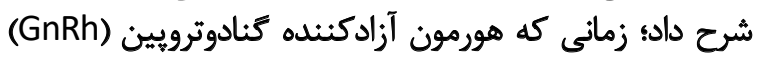

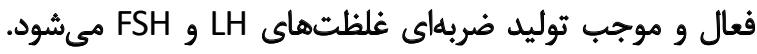

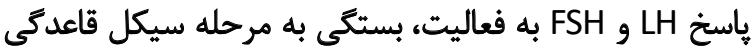

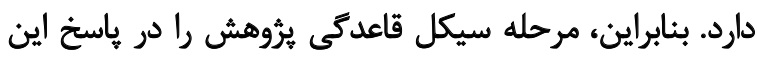

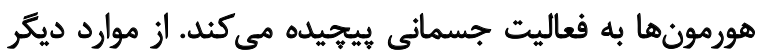

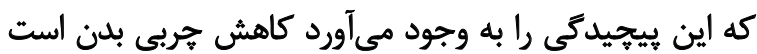

ورزش منظم موجب كاهش التهاب سيستميك در افراد سالهرو

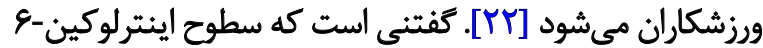

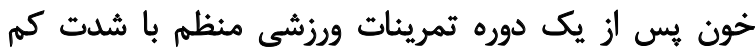

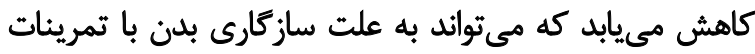

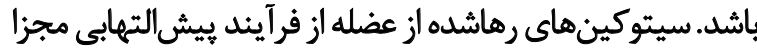

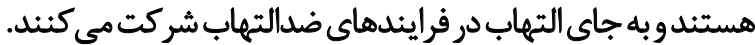

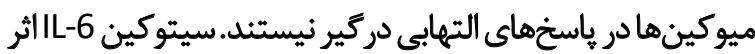

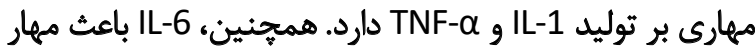
توليد TNF-

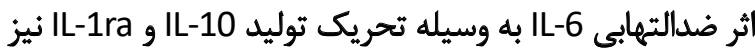

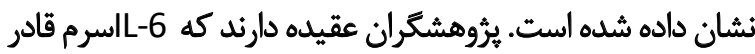

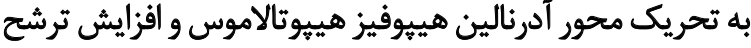

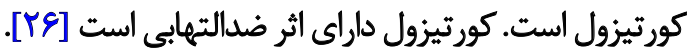

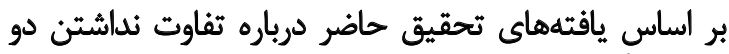

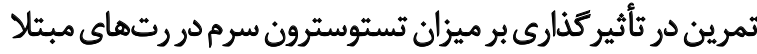

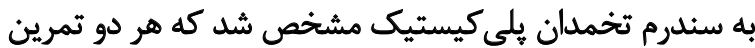

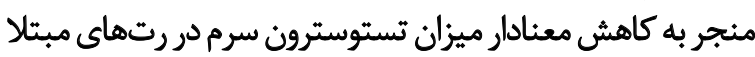

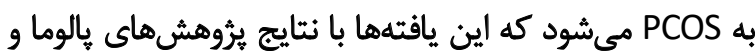

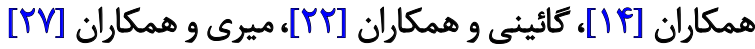

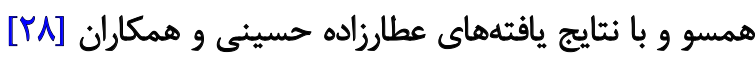
ناهمسيو بود.

ميرى و همكاران اثر شدت 1 هفته تمرين استقامتى شامل 9 


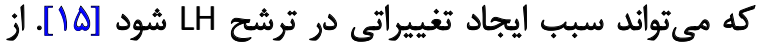

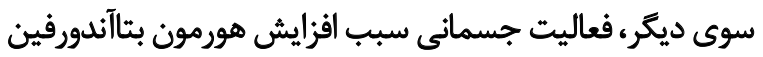

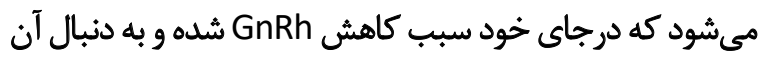

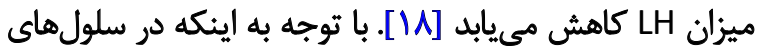

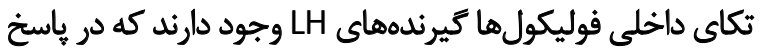

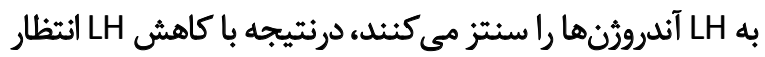

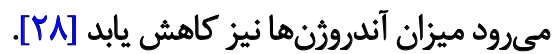

$$
\text { تثيجهُيرى }
$$

در كل نتيجه تحقيق نشان داد كه تمرين استقامتى در مقايسه

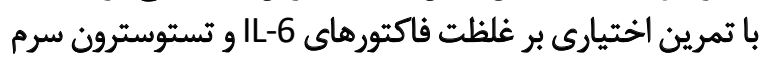

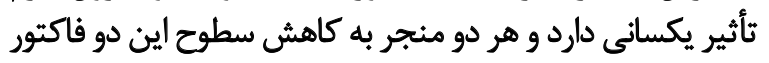

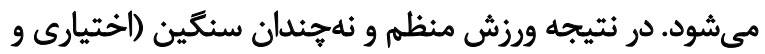

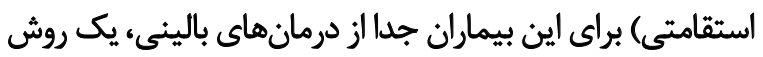

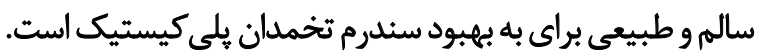

مالاحظات اخلاقي

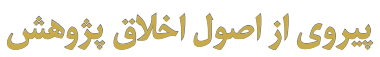

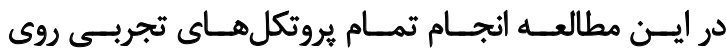

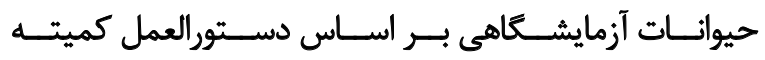

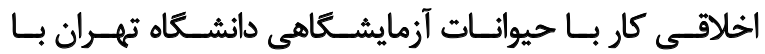

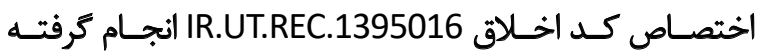

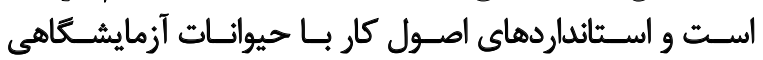

رعايست شــدند.

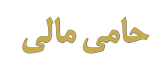

اين تحقيق هيج كونه كمك مالى از سازمان هاى تأمين مالى در

بخشهاى عمومى ، تجارى يا غيرانتفاعى دريافت نكردي الماني

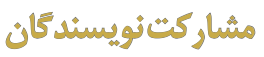

تمامى نويسندكان به يك اندازه در نكارش مقاله سهيم بودهاند.

$$
\text { تعارض منأقع }
$$

نويسندكان تصريح مي كنئ كه هيجگونه تضاد منافعى در

يرؤش حاضر وجود ندارد.

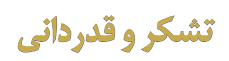

بدينوسيله از مسئولين و دستاندركاران محترم دانشكده تربيتبدنى دانشكاه تهران براي فراهم كردن مقدمات اندان انجام يرورثه

حاضر تشكر و قدرداني مي تشود. 


\section{References}

[1] Duleba AJ, Dokras A. Is PCOS an inflammatory process? Fertil Steril. 2012; 97(1):7-12. [DOI:10.1016/j.fertnstert.2011.11.023] [PMID] [PMCID]

[2] Ahmadi A, AghaAli Nejad H, Garehkhanloo R, Zarifi A. [Study of the relationship between IL- 6 changes (6) and serum Creatine Kinase (CK) in active girls after two sub intracranial activity and extrovert (Persian)]. Olympics. 2009; 17(2):63-72. https://www.sid.ir/fa/jour$\mathrm{nal} /$ ViewPaper.aspx?id=85524

[3] Nelson VL, Qin K-N, Rosenfield RL, Wood JR, Penning TM, Legro $\mathrm{RS}$, et al. The biochemical basis for increased testosterone production in theca cells propagated from patients with polycystic ovary syndrome. J Clin Endocrinol Metab. 2001; 86(12):5925-33. [DOI:10.1210/jcem.86.12.8088] [PMID]

[4] Baud V, Karin M. Signal transduction by tumor necrosis factor and its relatives. Trends Cell Biol. 2001; 11(9):372-7. [DOI:10.1016/S09628924(01)02064-5]

[5] Manneras L, Jonsdottir IH, Holmang A, Lonn M, Stener-Victorin E. Low-frequency electro-acupuncture and physical exercise improve metabolic disturbances and modulate gene expression in adipose tissue in rats with dihydrotestosterone-induced polycystic ovary syndrome. Endocrinology. 2008; 149(7):3559-68. [DOI:10.1210/ en.2008-0053] [PMID]

[6] Moro C, Pasarica M, Elkind-Hirsch K, Redman LM. Aerobic exercise training improves atrial natriuretic peptide and catecholamine-mediated lipolysis in obese women with polycystic ovary syndrome. J Clin Endocrinol Metab. 2009; 94(7):2579-86. [DOI:10.1210/jc.20090051] [PMID] [PMCID]

[7] Schulster A, Farookhi R, Brawer JR. Polycystic ovarian condition in estradiol valerate-treated rats: Spontaneous changes in characteristic endocrine features. Biol Reprod. 1984; 31(3):587-93. [DOI:10.1095/ biolreprod31.3.587] [PMID]

[8] 8. Dantas WS, das Neves W, Gil S, Barcellos CRG, Rocha MP, de SáPinto AL, et al. Exercise-induced anti-inflammatory effects in overweight/obese women with polycystic ovary syndrome. Cytokine. 2019; 120:66-70. [DOI:10.1016/j.cyto.2019.04.006] [PMID]

[9] Fatemi I, Pak-Hashemi M, Mohammadi-Nasab M, Kaeidi A, Hakimizadeh $E$, Hassanipour $M$. [The effects of endurance training in anxiety-like behaviors in rats with polycystic ovarian syndrome (Persian)]. J Jiroft Univ Med Sci. 2021; 7(4):498-505. https://journal.jmu.ac.ir/ browse.php?a_id=427\&sid=1\&slc_lang=en

[10] Halama A, Aye MM, Dargham SR, Kulinski M, Suhre K, Atkin SL. Metabolomics of dynamic changes in insulin resistance before and after exercise in PCOS. Front Endocrinol (Lausanne). 2019; 10:116. [DOI:10.3389/fendo.2019.00116] [PMID] [PMCID]

[11] Kraemer WJ, Fleck SJ, Deschenes MR. Exercise physiology: Integrating theory and application. Philadelphia: Lippincott Williams \& Wilkins; 2011. https://books.google.com/books/about/Exercise Physiology.html?id=RWP3JQfvHGEC

[12] Sirmans SM, Pate KA. Epidemiology, diagnosis, and management of polycystic ovary syndrome. Clin Epidemiol. 2014; 6:1-13. [DOI:10.2147/CLEP.S37559] [PMID] [PMCID]

[13] Palomba S, Giallauria F, Falbo A, Russo T, Oppedisano R, Tolino A, et al. Structured exercise training programme versus hypocaloric hyperproteic diet in obese polycystic ovary syndrome patients with anovulatory infertility: A 24-week pilot study. Hum Reprod. 2008; 23(3):642-50. [DOI:10.1093/humrep/dem391] [PMID]
[14] Aghaie F, Khazali H, Hedayati M, Akbarnejad A. The effects of moderate treadmill and running wheel exercises on oxidative stress in female rats with steroidinduced polycystic ovaries. Physiol Pharmacol. 2016; 20(4):277-86. https://iranjournals.nlai.ir/handle/123456789/511266

[15] Daryanoosh F, Jafari H, Rahimi E, Mehrbani D, Soltani F. The effect of eight week interval acute training on plasma visfatin, TNF-aand IL-6 in rats: A brief report. Tehran University Medical Journal. 2013 71(9):603-8. https://web.b.ebscohost.com/abstract?direct=true\&pr ofile=ehost\&scope=

[16] Thirunavukkarasu V, Balakrishnan S, Ravichandran M, Anuradha C. Influence of 6-week exercise training on erythrocyte and liver antioxidant defense in hyperinsulinemic rats. Comparative Biochemistry and Physiology Part C: Toxicology \& Pharmacology. 2003; 135(1):31-7. https://www.sciencedirect.com/science/article/abs/ pii/S1532045603000450

[17] Kenney WL, Wilmore JH, Costill DL. Physiology of sport and exercise. 6th ed. Champaign: Human kinetics; 2015. https://books.google.com/books/about/Physiology_of_Sport_and_Exercise_6th_Edi. html?id=OfjPoQEACAAJ

[18] Diane A, Pierce WD, Heth CD, Russell JC, Richard D, Proctor SD. Feeding history and obese-prone genotype increase survival of rats exposed to a challenge of food restriction and wheel running. Obesity (Silver Spring). 2012; 20(9):1787-95 [DOI:10.1038/oby.2011.326] [PMID]

[19] Azarian F, Farsi S, Hosseini SA, Azarbayjani MA. The effect of endurance training and crocin consumption on anxiety-like behaviors and aerobic power in rats with alzheimer's. Iran J psychiatry Behav Sci. 2019; 13(4):e89011. [DOI:10.5812/ijpbs.89011]

[20] Abdolmaleki F, Heidarianpour A. Endurance exercise training restores diabetes-induced alteration in circulating Glycosylphosphatidylinositol-specific phospholipase $D$ levels in rats. Diabetol Metab Syndr. 2020; 12:43. [DOI:10.1186/s13098-020-00553-z] [PMID] [PMCID]

[21] Gilani N, Zamani Rad F, Ebrahimi M, Haghshenas R. Effect of eight weeks endurance training on ovarian androgens in women with polycystic ovary syndrome: Application of multivariate longitudinal models. Int J Appl Exerc Physiol. 2019; 8(2.1):757-62. https://sciexplore.ir/Documents/Details/921-293-703-638

[22] Redman LM, Elkind-Hirsch K, Ravussin E. Aerobic exercise in women with polycystic ovary syndrome improves ovarian morphology independent of changes in body composition. Fertil Steril. 2011 95(8):2696-9. [DOI:10.1016/j.fertnstert.2011.01.137] [PMID] [PMCID]

[23] Papanicolaou DA, Wilder RL, Manolagas SC, Chrousos GP. The pathophysiologic roles of interleukin-6 in human disease. Ann Intern Med. 1998; 128(2):127-37. [DOI:10.7326/0003-4819-128-2 199801150-00009] [PMID]

[24] Xu X, Du C, Zheng Q, Peng L, Sun Y. Effect of metformin on serum interleukin-6 levels in polycystic ovary syndrome: A systematic review. BMC Womens Health. 2014; 14:93. [DOI:10.1186/1472-6874-14-93] [PMID] [PMCID]

[25] Pedersen BK, Febbraio MA. Muscle as an endocrine organ: Focus on muscle-derived interleukin-6. Physiol Rev. 2008; 88(4):1379-406. [DOI:10.1152/physrev.90100.2007] [PMID]

[26] Scheele C, Nielsen S, Pedersen BK. ROS and myokines promote muscle adaptation to exercise. Trends Endocrinol Metab. 2009, 20(3):95-9. [DOI:10.1016/j.tem.2008.12.002] [PMID] 
[27] Miri MS, Nikseresht A, Karimi Jashni $H$, Kargar Jahromi $H$, Sobhanian S. Effect of exercise on sex-hormone in rats with polycystic ovary syndrome. J Jahrom Univ Med Sci. 2013; 11(3):35-42. [DOI:10.29252/jmj.11.3.39]

[28] Attarzadeh Hosseini R, Ali Sardar M, Taghavi M, Yaz Khosh FT. [The effect of aerobic exercise program on the level of lutein hormones, follicular stimulants, testosterone and plasma dehydration in obese female women with polycystic ovary syndrome (Persian)]. Iran J Endocrinol Metab. 2012; 14(1):39-46. https://ijem.sbmu.ac.ir/article1-1080-fa.pdf 